\title{
Evaluation of the shedding routes and serological patterns in experimentally-induced Brucella melitensis infection in dexamethasone- treated and transport-stressed goats
}

\author{
Polycarp Nwunuji Tanko ${ }^{1}$, Benjamin O. Emikpe ${ }^{2}$ and Yusoff Mohd Sabri ${ }^{1}$
}

1. Department of Veterinary Pathology and Microbiology, Faculty of Veterinary Medicine, Universiti Putra Malaysia, Malaysia; 2. Department of Veterinary Pathology, Faculty of Veterinary Medicine, University of Ibadan, Ibadan, Nigeria Corresponding author: Yusoff Mohd Sabri, email: sabri@vet.upm.edu.my

Received: 12-03-2013, Revised: 11-05-2013, Accepted: 13-05-2013, Published online: 18-07-2013

doi: 10.14202 /vetworld.2013.686-692

How to cite this article: Tanko PN, Emikpe BO and Sabri MY (2013) Evaluation of the shedding routes and serological patterns in experimentally-induced Brucella melitensis infection in dexamethasone-treated and transport-stressed goats, Veterinary World 6(9): 686-692.

\begin{abstract}
Aim: To identify and evaluate the shedding routes and patterns following experimentally-induced Brucella melitensis infection in dexamethasone-treated and transport-stressed goats.

Materials and Methods: Twenty four healthy, adult goats were divided into 4 groups: A, B, C and D respectively. Group A was treated with dexamethasone for 8 days prior to inoculation with $10^{7}$ Colony Forming Units of B. melitensis via the intraocular route. Group B was transported for 3 hours prior to inoculation with a similar dose. Group C was inoculated with a similar dose without subjecting the animals to any prior treatment, and this group served as our positive control. Group D was not inoculated with the infective dose and served as our negative control. Blood samples along with nasal, ocular, and vaginal swabs were collected on days $0,3,7,10,14$, and weekly thereafter until day 63 post inoculation (pi) and were analyzed by PCR, Rose Bengal Plate Test (RBPT), and indirect ELISA techniques.

Results: The nasal, ocular and vaginal swabs tested positive for Brucellosis with PCR from day 7, with nasal route being the first and most consistent route to reveal the positive results. Group B showed the earliest onset of shedding the bacterium (day 7) followed by group A which started from day 10 and shed relatively more positive of the bacterium via the routes examined. Blood samples tested positive with PCR from day 7 through 14 and the results were inconsistent subsequently. Sera samples tested positive with RBPT on day 14 in all the 3 infected groups but more consistent in group C. On the other hand, tests using ELISA showed positive results from day 7 pi, with group C having a 100\% seroconvertion -while groups A and B showed only $50 \%$ seroconvertion.
\end{abstract}

Conclusion: The consistent shedding via the nasal, ocular, and vaginal routes in groups A and B implied possible immunosuppression in the infected animals. We recommend that programs designed to control Brucellosis should consider analyzing a larger number of biological samples to enhance the accuracy of identification of shedders.

Keywords: brucellosis, consistent shedding, goats, immunosuppression, polymerase chain reaction, serological tests, swabs.

\section{Introduction}

Brucellosis is now recognized as one of the most important among the common zoonotic diseases across the world and is a disease that poses a major threat to both human health and animal populations [1]. The World Health Organization considers it as a neglected zoonosis, because adequate control programmes were not planned in numerous countries despite its huge impact not only on human and animal health, but also on the economy [2]. Brucellosis, especially affecting the food animals, poses a barrier to the trade of animals and animal products between countries. This is because Brucellosis causes considerable economic losses due to abortions and fertility problems, encountered often in the sheep and goat industry $[3,4,5]$. Brucellosis in small ruminants, especially goats is caused by $B$. melitensis [6] and the incidences of transmission to the human population have significantly increased in most

This article is an open access article licensed under the terms of the Creative Commons Attribution License (http://creativecommons. org/licenses/by/2.0) which permits unrestricted use, distribution and reproduction in any medium, provided the work is properly cited. of the developing nations that have no effective vaccination-based control and slaughter programmes, such as the countries in Mediterranean, Middle East, Africa, Latin America, and parts of Asia [7].

Goats in the tropics are usually reared under the traditional extensive management system and are often transported over a considerable distance for marketing and slaughter purposes $[8,9]$. This long range transportation, an age old inevitable husbandry practice [10], creates undue stress on the animals. In this regard, it is important to note that the artificially-induced stress upon administration of dexamethasone has been reported to mimic the stress elicited by natural causes $[11,12,13]$. Studies investigating the effect of stress on the productivity of small ruminants' have primarily focused on caprine pneumonia $[14,15]$ with very little emphasis on the elucidating the effect of stress on the transmission of zoonotic diseases.

With a myriad of stress factors that goats are constantly subjected to, it is imperative to understand the interactions between stress, reproductive zoonotic disease occurrence, and transmission of the disease. 
Understanding the interaction between the aforementioned factors is critical to enhance our knowledge base for proper planning and swift control of these diseases in goats, especially in those herds that are constantly subjected to environmental and managemental stresses. To the best of our knowledge, there is no study that evaluated the relationship between stressful conditions and the shedding pattern of Brucella melitensis infection in goats.

Therefore, this study seeks to elucidate the shedding routes of experimentally-infected goats by subjecting them to dexamethasone treatment and transport stress in order to evaluate the shedding routes and patterns using serological tests and PCR of samples collected from potential shedders.

\section{Materials and Methods}

Animals: Twenty four healthy boar goats used for this experiment were housed in designated pens in the Faculty of Veterinary Medicine, experimental unit, Universiti Putra Malaysia. The animals were selected after their sera tested negative for Brucellosis using Rose Bengal Plate Test (RBPT) and indirect ELISA. Additionally, the ocular, nasal and vaginal swabs from these goats were plated in Brucella agar and incubated at $37^{\circ} \mathrm{C}$ for 4 days. The plates were confirmed negative using PCR using specific primers to B. melitensis. The animals were fed with concentrates, grass and water ad libitum. Animals were stabilized for 3 weeks and then randomly divided into 4 groups designated as A (6), B (6), C (6) and D (6) prior to the onset of the experiment. Animals in group A were administered dexamethasone (Troy Laboratories Pvt. Limited, Australia) @2mg/ $10 \mathrm{~kg}$ body weight for eight days following which they were infected with $10^{7}$ Colony Forming Units (CFU) of B. melitensis strain 183. Animals in group B were transported by road. The mean environmental temperature ranged between $36-40^{\circ} \mathrm{C}$ (Average $38^{\circ} \mathrm{C}$ ) and the relative humidity was between $17-20 \%$.

Ethical approval: The experimental design and animal treatment was in compliance with the humane methods recommended by the Research Ethics Committee, Faculty of Veterinary Medicine, Universiti Putra Malaysia (AUPNo: 12R159).

The handling, loading and transportation of the experimental goats were carried out humanely in accordance with the guidelines governing animal transport welfare by road [16]. A standard Bedford, open-roof (Ford, UK), vehicle was used for the transportation. The floor of the van was provided with beddings (wood shavings) for secure footing. The goats were stocked at a rate of $0.2 \mathrm{~m}^{2}$ per animal [17]. The journey commenced by 1000 hours and was terminated at 1330 hours. The vehicle travelled on a typical asphalt double lane road from Serdang to Malaka and back $\left(3^{\circ} 00^{\prime} \mathrm{N}, 101^{\circ} 40^{\prime} \mathrm{E}\right)$ Malaysia, covering a total distance of about $350 \mathrm{~km}$. The vehicle travelled with an average speed of $60 \mathrm{~km} / \mathrm{h}$ and had a stop-over for less than 15 minutes under a shade (for resting purposes). The goats were neither fed nor watered during the resting period. The animals were then infected, after being off loaded, with doses that were similar to those administered to group A. Animals in group $\mathrm{C}$ were neither treated with dexame-thasone nor transported but were also infected with doses similar to those administered to group A and served as positive controls. Animals in group D served as the negative controls.

Sampling: Blood, ocular, nasal and vaginal swabs were collected on days $0,3,7,10,14$ and weekly thereafter until day 63 post infection (pi). The blood samples were collected in duplicates containing both heparinized and non-heparinized samples. Dry and sterile cotton wool swabs were used to collect the ocular, nasal and vaginal swabs.

PCR assay: DNA was extracted from the heparinized blood using Promega kit by following the manufacturer's instructions after which PCR was performed using $B$. melitensis specific primers [18] ( $5^{1}$ AGTGTTTCG GC TCAGAATTAATC $3^{1}$ and $5^{1}$ CATGCGCTAT GTCT G GTTAC $3^{1}$ ). Briefly, the PCR was carried out with $2 \mu 1$ of the extracted DNA in $48 \mu \mathrm{l}$ of master mix (Fermentas, Germany) in a total volume of $50 \mu 1$ in Eppendorf master cycler (Germany). The Eppendorf master cycler was programmed as follows; Initial denaturation at $94^{\circ} \mathrm{C}$ for 4 minutes followed by 34 cycles at $94^{\circ} \mathrm{C}$ for 1 minute annealing at $65.75^{\circ} \mathrm{C}$ for 1 minute, extension at $72^{\circ} \mathrm{C}$ for 1 minute, final extension at $72^{\circ} \mathrm{C}$ for 10 minutes and hold at $4^{\circ} \mathrm{C}$. The PCR products were analyzed by electrophoresis on $1 \%$ agarose gel stained with gel red and visualized and photographed.

The swabs were plated on Brucella Agar and incubated at $37^{\circ} \mathrm{C}$ for 4 days after which they were tested using PCR with $B$. melitensis primers as stated earlier. Briefly, $2 \mu 1$ of DNAZol was added to $2 \mu 1$ of the colonies in Eppendorf tubes and allowed to stay for 15 minutes before adding the master mix as described above. The negative control used was the master mix without DNA template and the external positive control was DNA templates extracted from a Brucella broth containing $10^{7} \mathrm{CFU}$ of B. melitensis strain 183 using Promega Genomic DNA purification kit (Promega, Germany).

Sera were also harvested from the nonheparinized blood and stored at $-20^{\circ} \mathrm{C}$ until used for RBPT and ELISA. For the RBPT, a drop from each serum sample was laid on a plate and a drop of the RBPT reagent was added, mixed well and allowed to stay for 10 minutes. In this test, a classical agglutination reaction was considered positive for Brucella. For the indirect ELISA, the antigen was prepared and used to coat the wells of a polystyrene plate (Nunc-Immuno plate with MaxiSorp surface). After overnight incubation at $4^{\circ} \mathrm{C}$, the plate was washed 3 times with the washing 
Table-1. PCR result for Nasal, Ocular and Vaginal swabs that were tested for B. melitensis.

\begin{tabular}{llllllllllll}
\hline Group & Shedding Route & $\mathbf{D 7}$ & $\mathbf{D 1 0}$ & $\mathbf{D 1 4}$ & $\mathbf{D 2 1}$ & $\mathbf{D 2 8}$ & $\mathbf{D 3 5}$ & $\mathbf{D 4 2}$ & $\mathbf{D 4 9}$ & $\mathbf{D 5 6}$ & $\mathbf{D 6 3}$ \\
\hline A & Nasal & 0 & 0 & $+(2)$ & $+(3)$ & $+(2)$ & $+(2)$ & $+(1)$ & 0 & 0 & 0 \\
& Ocular & 0 & $+(1)$ & $+(1)$ & $+(2)$ & 0 & $+(2)$ & $+(2)$ & 0 & 0 & $+(1)$ \\
& Vagina & 0 & $+(2)$ & $+(1)$ & $+(3)$ & 0 & $+(1)$ & 0 & 0 & 0 & 0 \\
B & Nasal & $+(3)$ & $+(1)$ & 0 & 0 & 0 & $+(2)$ & $+(1)$ & 0 & $+(1)$ & 0 \\
& Ocular & 0 & $+(2)$ & $+(1)$ & $+(1)$ & $+(1)$ & 0 & 0 & $+(1)$ & 0 & 0 \\
& Vagina & 0 & $+(1)$ & 0 & 0 & 0 & 0 & 0 & 0 & 0 & 0 \\
C & Nasal & 0 & $+(1)$ & $+(1)$ & $+(1)$ & 0 & $+(1)$ & 0 & 0 & $+(1)$ & 0 \\
& Ocular & 0 & 0 & 0 & 0 & 0 & 0 & 0 & 0 & 0 & 0 \\
& Vagina & 0 & 0 & 0 & 0 & 0 & $+(1)$ & $+(1)$ & $+(1)$ & 0 & 0 \\
D & Nasal & 0 & 0 & 0 & 0 & 0 & 0 & 0 & 0 & 0 & 0 \\
& Ocular & 0 & 0 & 0 & 0 & 0 & 0 & 0 & 0 & 0 & 0 \\
& Vagina & 0 & 0 & 0 & 0 & 0 & 0 & 0 & 0 & 0 & 0 \\
\hline
\end{tabular}

Table-2. Comparison of the relationship between the 3 shedding routes

\begin{tabular}{|c|c|c|c|c|c|c|c|c|}
\hline Group & Shedding route & D7 & D10 & D14 & D21 & D28 & D42 & D49 \\
\hline \multirow[t]{4}{*}{ A } & NV & 0 & 0 & $+(1)$ & $+(2)$ & 0 & $+(1)$ & 0 \\
\hline & NO & 0 & 0 & 0 & $+(1)$ & 0 & $+(2)$ & $+(1)$ \\
\hline & OV & 0 & $+(1)$ & 0 & $+(2)$ & 0 & $+(1)$ & 0 \\
\hline & NOV & 0 & 0 & 0 & $+(1)$ & 0 & $+(1)$ & 0 \\
\hline \multirow[t]{4}{*}{ B } & NV & $+(1)$ & 0 & 0 & 0 & 0 & $\begin{array}{l}+(1) \\
\end{array}$ & 0 \\
\hline & NO & 0 & 0 & $+(1)$ & 0 & 0 & 0 & 0 \\
\hline & OV & 0 & 0 & 0 & 0 & 0 & 0 & 0 \\
\hline & NOV & 0 & 0 & 0 & 0 & 0 & 0 & 0 \\
\hline \multirow[t]{4}{*}{ C } & NV & 0 & 0 & 0 & 0 & 0 & 0 & 0 \\
\hline & NO & 0 & 0 & 0 & 0 & 0 & 0 & 0 \\
\hline & OV & 0 & 0 & 0 & 0 & 0 & 0 & 0 \\
\hline & NOV & 0 & 0 & 0 & 0 & 0 & 0 & 0 \\
\hline
\end{tabular}

$\mathrm{N}$ : Nasal, V: Vagina and O: Ocular route

buffer (Phosphate Buffered Saline, pH 7.4, with 0.05\% Tween 20). $200 \mu 1$ of Blocking Buffer was added to each well and incubated at $37^{\circ} \mathrm{C}$ for $1 \mathrm{hr}$ and washed 3 times again with the washing buffer. $50 \mu \mathrm{l}$ of serum from samples at the dilution of 1:200 were added to the wells and incubated for another $1 \mathrm{hr}$ and washed 3 times with the washing buffer. Horse Radish Rabbit AntiGoat immunoconjugate IgG Peroxidase (Nordic Immunology, Netherlands) was added and incubated for another hour and washed again 3 times. Then $100 \mu \mathrm{l}$ of 3,3,5,5-tetramethylbenzidine (TMB) One solution substrate (Promega, USA) was added and incubated for 10 minutes. The reaction was stopped by the addition of $50 \mu 1$ of $2.5 \mathrm{M}$ sulphuric acid. The optical density values were measured at $450 \mathrm{~nm}$ wavelength in an AnthosZenyth 340 st reader (Austria).

Statistical analysis: Data obtained in this study were analyzed using Statistix 9.0 software, using repeated Measure Analysis of Variance and LSD. All-pairwise comparisons was used to test the differences between the groups. The criterion for statistical significance was set at $P<0.05$.

\section{Results}

General observations: Group A had a consistently higher body temperature when compared to groups B and $\mathrm{C}$. The thermal response was undulant and differed significantly between groups. There was no significant difference in the body temperature pattern in groups $\mathrm{A}$ and $\mathrm{B}$, but there was a significant difference between groups $\mathrm{A}, \mathrm{B}$ and $\mathrm{C}$ when compared to group D. A generalized inappetence was observed in all the infected groups on day 8 pi, but more severe in group A.
Sneezing, ear biting and coughing were also observed to varying degrees within the first 21 days pi in groups A and Bonly.

Shedding route: The animals started shedding the bacterium from day 7 pi, first in group B with $50 \%$ of the animals shedding through the nasal route followed by the vaginal route (Table-1). Significant difference $(P<0.05)$ in the shedding pattern was observed in groups A and B when compared to group C. Group A started shedding through the nasal route from day 10 pi. As the infection progressed, majority of the animals in group A shed the bacterium via all the routes examined and relatively for a longer period with some of the animals shedding consistently for 5 weeks. Group C, however, did not shed the bacterium via the vaginal route until day $35 \mathrm{pi}$; while groups $\mathrm{A}$ and $\mathrm{B}$ shed through this route as early as day $10 \mathrm{pi}$. Throughout the experiment, only 1 animal from A shed the bacterium in all the 3 routes at day 21 and day 35 . By day 14 pi, $100 \%$ of animals in groups A and B shed the bacterium at least once -while only $33 \%$ of animals in group C shed the bacterium. All the animals shed the bacterium at least once in the period covered for the experiment with the exception of the negative control group (group D) which remained negative throughout since group D was not inoculated with the infective dose.

Comparison of the three shedding routes: Groups $\mathrm{A}$ and $\mathrm{B}$ shed significantly $(P<0.05)$ higher when compared to group C. $100 \%$ of the animals in group A shed the bacterium in at least 2 routes at a time in this study and they shed more consistently when compared to groups B and C (Table-2). In group A, 50\% of the animals shed concomitantly through the nasal and 


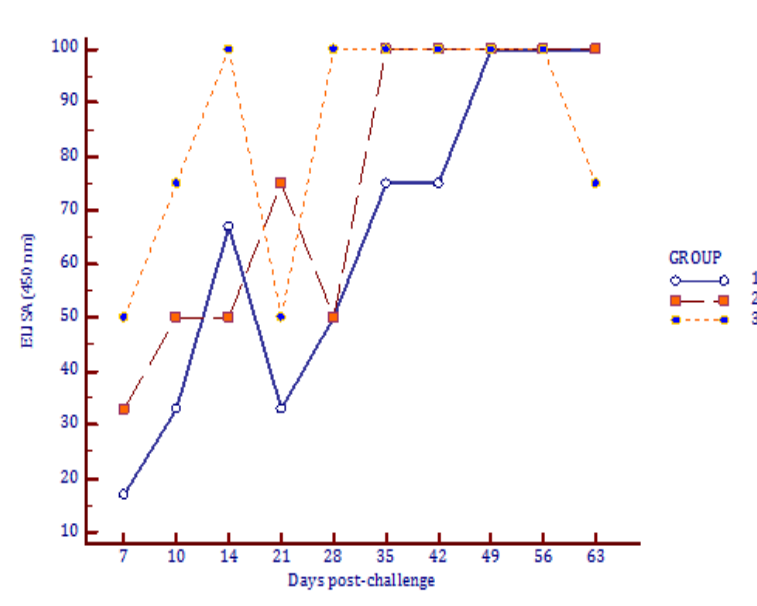

Figure-1. The pattern of antibody response to experimental $B$. melitensis infection in group A : $1, B: 2$ and $C: 3$; in percentage of positivity

vaginal routes, $33 \%$ through nasal and ocular routes, $50 \%$ through ocular and vaginal and $17 \%$ shed twice via all the 3 routes.

In group $\mathrm{B}$, however, only $33 \%$ of the animals shed the bacterium through at least 2 routes at a time. $33 \%$ of the animals shed through the nasal and ocular routes and $17 \%$ shed through nasal and ocular routes while none shed through the ocular and vaginal and all the 3 routes. None of the animals from group $\mathrm{C}$ shed the bacterium in any 2 routes at a time throughout the study. Even though the ocular route was used for the infection, none of the animals from group $\mathrm{C}$ shed through this route. However, none of the animals from all the groups shed the bacterium in any 2 routes at a time after day 49 and also the consistency of shedding decreased after day 49.

Serological tests: For the RBPT, Group C tested positive significantly $(P<0.05)$ when compared to groups A and B on day 14 pi, where $100 \%$ of animals in group $\mathrm{C}$ tested positive and the number of positives eventually dropped and remained constant at $50 \%$ from day 21 to day 49 pi. $60 \%$ of those in group A tested positive at day 14 and the number of positives also dropped to $17 \%$ from day 21 onward. $50 \%$ of animals from group B tested positive on day 14 and then dropped to $17 \%$ from day 21 onward. The indirect ELISA (Figure-1) was able to detect the antigen earlier compared to the RBPT in this study. $50 \%$ of the animals in group $\mathrm{C}$ tested positive at day 7 pi and by day 14 pi, all the animals in this group tested positive. $33 \%$ of the animals in Group B tested positive on day 7 pi and by day $42 \mathrm{pi}, 100 \%(6 / 6)$ tested positive and remained positive throughout the study. Only $17 \%$ of the animals in group A tested positive on day 7 pi but $100 \%$ by day 49 pi and remained positive throughout the study. There was a significant difference $(P<0.05)$ in the antibody titres among the treatment groups from day 7 through day 42 pi.

Relationship between the shedding routes and the serological response: In this study, the stressed animals showed increased shedding of the bacterium and in at

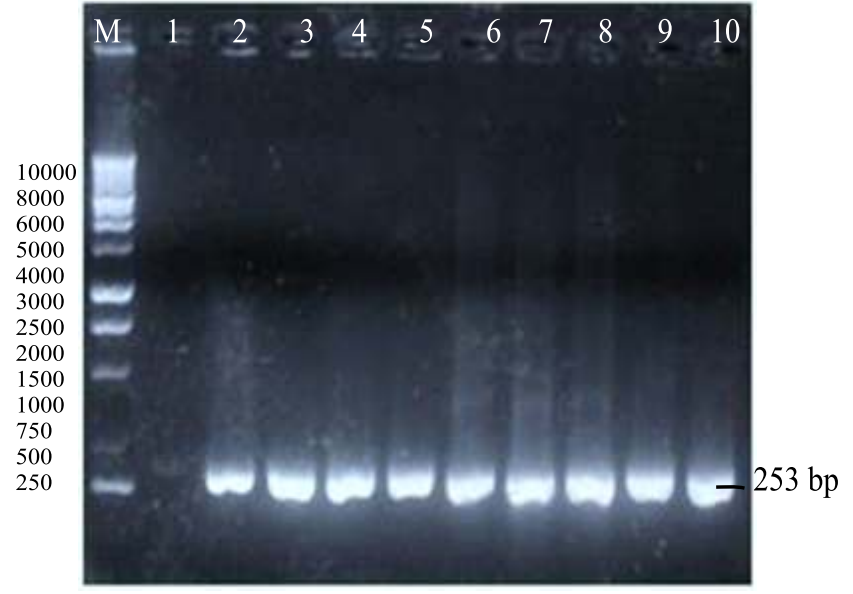

Figure-2. PCR of blood samples on day 7 pi. M: 1-kb standard ladder, Lane 1: negative control, Lane 2: positive control, Lane 3-10: positive samples

least 2 out of the 3 routes investigated, but the serological tests (RBPT and iELISA) were not consistent in identifying all the shedders. Animals in group A started shedding on day $10 \mathrm{pi}$, where $33 \%$ of the animals shed the bacterium. These shedders were positive with ELISA(ELISA been more sensitive), but negative with RBPT. However, the RBPT was able to identify all the shedders at day 14 pi and by day $21 \mathrm{pi}$, which was the peak shedding period (100\%) for group A, both ELISA and RBPT were able to detect only $17 \%$ of the animals in group $\mathrm{A}$ that were shedding the bacterium. Even though the ability of the serological tests to identify the shedders increased over time in this study, they were not able to detect all the shedders from this group. There was no significant difference in the ability of RBPT and ELISA in the detection of shedders and nonshedders among the group. On the other hand, PCR was able to detect more accurately in groups $\mathrm{A}$ and $\mathrm{B}$ than group $\mathrm{C}$ in all the routes with significant difference $(P<$ 0.05 ) between groups $\mathrm{A}$ and $\mathrm{B}$ compared to group $\mathrm{C}$ with respect to the ocular route.

Animals in group B however, started shedding as early as day 7 pi and unlike group A, peaked between day 7 and day 10 pi with $50 \%$ of the animals shedding. ELISA was able to detect only $25 \%$ of the shedders at this period, while RBPT was negative in all. Subsequently, the rate of shedding dropped to $17 \%$ at day 14 and ELISA was able to detect all the shedders while the ability of RBPT to detect the positives was relatively less. We also observed that the ELISA was able to identify more number of the shedders over time. Animals from group $\mathrm{C}$ shed less of the bacterium compared to groups A and B, as expected. By day 14, none of the animals in group $\mathrm{C}$ was shedding via the routes examined even though some serologically tested positive with both ELISA and RBPT.

Polymerase Chain Reaction (PCR) was carried out using DNA extracted from the blood samples of the animals and this test detected positive animals earlier than the serological tests. PCR detected $100 \%$ of the animals in group $\mathrm{C}$ and $50 \%$ in group B as early as day 3 
pi. By day 7 pi, blood samples from all the infected animals tested positive and thereafter, most of the animals were negative. However, group D (the uninfected control group) remained negative throughout the study.

\section{Discussion}

The shedding of $B$. melitensis by domesticated animals poses a very serious public health risk. Infected animals are often the source of human infection with brucellosis, especially domesticated animals and the control of this disease in humans is only achievable through the effective control of the disease in animals, hence its public health significance. Most Control programmes for Brucellosis often rely on serological tests for screening and/or identification of infected animals without any emphasis on the detection of apparently animals that are shedding the bacterium. This study evaluates the shedding routes and the diagnostic potential of analyzing more than one biological sample in experimentally-infected (dexamethasone-treated or transport-stressed) goats as evaluated for Coxiella burnetti infection in the earlier studies $[19,20]$.

All the infected animals in this study shed the bacterium at least once via one of the 3 routes investigated, the earliest being the nasal route from the transport-stressed group (group B) at day 7 pi while those belonging to the dexamethasone-treated group (group A) shed more consistently in all the routes examined and for a longer period starting from day 10 pi when compared to animals that were infected without any prior stress-inducing treatments (group C). This result is in accord with earlier studies [21, 22, 23] which reported that stress factors are linked to increased pathogen carriage, increased disease susceptibility, and possible pathogen shedding. Thus, stress factors can lead to immune dysregulation [24]. There is evidence to show that immunosuppression could account for severe lesions being encountered in the respiratory tract in diseases such as peste des petits ruminants [13], and this may be one of the reasons why we observed the dominance of the nasal route in the shedding pattern in our study. The proximity and the linkage of the ocular and the nasal routes could also explain the dominance of the nasal route in shedding the bacteria.

The earlier onset and prolonged shedding of the bacterium by the stressed and infected animals in this study further confirmed that the combination of infection and stress could pose a very serious risk to the public health. Our finding also indicates that there is a high potential for the spread of this infection to humans who are involved in small ruminant husbandry practices, especially in the tropics. In agreement with our findings, earlier studies $[25,26]$ reported that Brucella infected animals could serve as sources of infection to other non-infected animals as well as the human populace. The relatively increased shedding of the bacterium through the nasal, ocular and vaginal routes in the stressed groups vs. the control animals (group C -infected, but not subjected to prior stress) further implies that stress could induce pathogen redistribution within the system of the infected animals and thereby constituting a high public health risk. This is typified by the earlier onset of shedding via the vaginal route as observed in the stressed groups (groups A and B), when compared to the unstressed group (group C), despite the use of intraocular route for the inoculation purpose. The peak shedding observed between days 7 and $10 \mathrm{pi}$ in group B showed the critical period at which the transported animals could shed; this period should be taken into account while devising the control strategies. The shedding observed in group A showed that chronically-stressed or immunosuppressed goats could continually shed the bacterium for considerably a longer period as observed in diseases such as Q-fever and enteric infections [19,22].

Our study also showed that the shedding rate in group $\mathrm{C}$ was relatively lower and inconsistent (Table-1). The increase in the number of shedding routes in the stressed animals, as reported in this study, further demonstrates the important role of stress in inducing not only the bacterial redistribution in vivo but also in the disease transmission.

In this study, the two serological techniques employed showed that stress induced either by dexamethasone or by transport is capable of causing immune dysregulation $[13,24]$, an affect that possibly can lead to less seroconvertion observed in the stressed groups showing only $50 \%$ of the animals being seropositive -while group C showed a 100\% (6/6) seroconvertion within the same period. The use of the serological techniques to identify potential shedders, especially in stressed goats, were not encouraging as our study showed that RBPT identified all the animals (100\%) shedding the bacterium in group $\mathrm{C}$ whereas only $66 \%$ and $50 \%$ of the animals from groups A and B tested positive, respectively. The indirect ELISA used in this study was able to detect positive animals at an earlier time when compared to the RBPT but it could not identify all the shedders in the course of this study until day 35. The reduced and inconsistent serological responses observed in groups A and B as detected with indirect ELISA and RBPT could possibly be assigned to stress-induced immune dysregulation [13].

The PCR performed using the DNA extracted from the blood samples identified the infected animals as early as day 3 pi, and on this day all the animals in group C tested positive $50 \%$ from group B and none from group A tested positive. By day 7 pi, all the blood samples of the animals infected in this study tested positive with PCR. Subsequently, the animals tested positive only sporadically. This could be attributed to the fact that the bacterium could have migrated from the blood and get trapped within the macrophages and non-professional phagocytes [27]. Furthermore, it was speculated that the inconsistency in the positive results could be due to re-infection originating from intermittent shedders. 


\section{Conclusion}

Our study provides vital information on the role of stress in modulating the pattern and routes of shedding of B. melitensis in dexamethasone-treated and transport-stressed goats. Our significant findings regarding the shedding patterns and the transmission of brucellosis in goats call for actions to alleviate the serious public health risks imposed by the shedding of Brucella by the infected goats. Based on our findings, we suggest that strategies aimed for controlling brucellosis should consider collecting and analyzing more than just one biological sample. Furthermore, we recommend that the control strategies should give due importance to various methods for the detection of persistent and intermittent shedders rather than just relying on serological tests to identify the infected animals.

\section{Authors' contributions}

PNT carried out the experiments and collected the samples. BOE carried out the data analysis. MYS was a project leader and carried out the data analysis. All authors drafted and revised the manuscript. All authors read and approved the final manuscript.

\section{Acknowledgements}

The authors remain grateful to all the staff of the histopathology laboratory, Necropsy building complex of the faculty of Veterinary medicine, Universiti Putra Malaysia. This project was funded by the Ministry of Science, Technology and Innovation (MOSTI), Malaysia under the Science Fund 5450546.

\section{Competing interests}

The authors declare that they have no competing interests.

\section{References}

1. Seleem, M.N., Boyle, S.M. and Sriranganathan, N. (2010) Brucellosis: A re-emerging zoonosis. Vet Microbiol. 140: 392-398.

2. Holt, H.R., Eltholth, M.M., Hegazy, Y.M., El-Tras, W.F., Tayel, A.A. and Guitian, J. (2011) Brucella spp. infection in large ruminants in an endemic area of Egypt: cross-sectional study investigating seroprevalence, risk factors and livestock owner's knowledge, attitudes and practices (KAPs). BMC Public Health, 11:341 doi:10.1186/1471-2458-11-341.

3. Aldomy, F., N. O. Hussein, L. Sawalha, K. Khatatbeh, and A. Aldomy. (2009) "A national survey of perinatal mortality in sheep and goats in Jordan," Pak Vet J, 29(3):02-106.

4. Samadi, M., Ababneh, M. M., Giadinis, N.D. and Lafi, S. Q. (2010) Ovine and Caprine Brucellosis (Brucella melitensis) in Aborted Animals in Jordanian Sheep and Goat Flocks. SAGE-Hindawi Access to Res Vet Med Int. Article ID 458695, 7 pages doi:10.4061/2010/458695.

5. Godfroid,J., Scholzc, H.C., Barbierd, T., Nicolasd, C., Wattiaue, P., Fretine, D., Whatmoref, A.M., Cloeckaertg, A., Blascoh, J.M., Moriyoni, I., Saegermanj, C., Mumak, J.B., Dahouk, A.S., Neubauern, H. and Letessond, J.J. (2011) Brucellosis at the animal/ecosystem/human interface at the beginning of the 21 st century. Prev. Vet. Med. doi:10.1016/j. prevetmed.2011.04.007.

6. Megid, J., Mathias L. A. and Robles, C.A. (2010) Clinical Manifestations of Brucellosis in Domestic Animals and Humans. The Open Vet Sci J. 4, 119-126.
7. Belay, T. (2008) Development of a real-time PCR assay for diagnosis of Brucella melitensis infection in sheep. University Putra Malaysia Institutional repository, 2008

8. Ayo, J.O. and Oladele, S.B. (1996) Natural antioxidants and their potential uses in prophylactics and therapy of disease conditions. W. Af. J. Pharmacol. Drug Res., 12: 69-76.

9. Minka, N.S. and Ayo, J.O. (2007) Physiological responses of transported goats treated with ascorbic acid during the hotdry season. Anim. Sci. J., 78: 164-172.

10. Fazio, E and Ferlazzo, A. (2003) Evaluation of stress during transport. Vet Res Com, 27(supplement 1): 519-524.

11. Zamri-Saad, M., Effendy, W. M., Maswati, M. A., Salim, N. and Sheikh-Omar, A. R. (1996) The goat as a model for studies of pneumonic pasteurellosis caused by Pasteurella multocida. Br. Vet. J., 152: 453-458.

12. Forsberg, N.E. (2004) Recent Insights Into Ruminant Immune Function: Effects of Stress and Immunostimulatory Nutritional Products. Florida Ruminant Nutrition Symposium.

13. Jagtap, S.P., Rajak, K.K., Garg, U.K., Sen, A.,Bhanuprakash, V., Sudhakar, S.B., Balamurugan, V., Patel, A., Ahuja, A., Singh, R.K. and Vanamayya, P.R. (2012) Effect of immunosuppression on pathogenesis of peste des petits ruminants (PPR) virus infection in goats, Microb Pathogen. Doi:10: 1016/j.micpath.2012.01.003.

14. Zamri, Saad, Jasni, M. S., Nurida, A. B. and Sheikh-Omar, A. R. (1991) Experimental infection of dexamethasone treated goats with Pasteurella haemolytica a2 Br. Vet. J. 147:5 65.

15. Mohamed, R. A. (2002) The effect of iron compounds and other factors on the pathogenesis of pneumonic pasteurellosis in Nubian goats. Ph.D. Thesis, Faculty of Veterinary Science, University of Khartoum, Sudan.

16. Richardson, C. (2002) Lowering stress in transported goats. Ontario Ministry of Agriculture and Food-Livestock Technology.Branch Northern Ontario Regional Office.

17. Zulkifli, Idrus., Bahyuddin, N., Wai, C. Y., Farjam, A. S., Sazili, A. Q., Rajion, M. A. and Meng, G. Y. (2010) Physiological responses in goats subjected to road transportation under the hot, humid tropical conditions, Int. J. Agric. Biol., 12: 840-844. Available online at 1814-9596 10-117/MSA/ 2010/12-6-840-844.

18. Redkar, R., Rose, S., Bricker, B. and Del Vecchio, V. (2001) Real-Time Detection of Brucella Abortus, Brucella Melitensis and Brucella Suis. Molecular and Cellular Probes, 15: 43-52.

19. Guatteo, R., Joly, A. and Beaudeau, F. (2012) Shedding and serological patterns of dairy cows following abortion associated with Coxiella burnetti DNA detection. Vet. Microbiol, 155 430-433.

20. Rousset, E., Berri, M., Durand, B., Dufour, P., Prigent, M., Delcroix, T., Touratier, A. and Rodolakis, A. (2009) Coxiella burnetii shedding routes and antibody response after outbreaks of $\mathrm{Q}$ fever induced abortion in dairy goats herds. Appl. Environ. Microbiol. 75(2):428. doi:10.1128/ AEM. 00690-08.

21. Burkholder, K.M., Thompson, K.L., Einstein, M.E., Applegate, T.J., Patterson, J.A. (2008) Influence of stressors on normal intestinal microbiota, intestinal morphology, and susceptibility to Salmonella enteritidis colonization in broilers. Poult. Sci. 87: 1734-1741.

22. Rostagno, M.H. (2009) Can stress in farm animals increase food safety risk? Foodborne Pathog. Dis. 6: 767-776.

23. Verbrugghe, E., FilipBoyen, Gaastra, W., Bekhuis, L., Leyman, B., Parys, A.V., Haesebrouck, F. and Pasmans, F. (2012) The complex interplay between stress and bacterial infections in animals. Vet Microbiol, 155:115-127.

24. Glaser, R., Yanga, E.V., Kimb, S., Donovanb, E.L., Chenb, M., Grossb, A.C., Webster, J.I., Marketonb, D.E., Sanford, H. and Barskyc, F. (2009) Norepinephrine upregulates VEGF, IL-8, and IL-6 expression in human melanoma tumor cell lines: Implications for stress-related enhancement of tumor progression. Brain, Behavior and Immunity, 23(2): 267-275.

25. Benkirane, A. (2006) "Ovine and caprine brucellosis: world 
distribution and control/eradication strategies in West Asia/North Africa region," Small Ruminant Res, 62(1-2): 19-25.

26. Hegazy, Y.M., Moawad, A., Osman, S., Ridler, A. and Guitian, J. (2011) Ruminant brucellosis in the Kafr El Sheikh governorate of the Nile Delta, Egypt: Prevalence of neglected zoonosis. PLoS Negl Trop Dis. 5(1):e944.

27. Xavier, M.N., Paixão, T.A., den Hartigh, A.B., Tsolis, R.M. and Santos, R.L. (2010) Pathogenesis of Brucella spp. The Open Vet. Sci. J., 4:109-118.

$* * * * * * * *$ 